\title{
ON GRAPHS WITH NO PROPER PERFECT DOMINATING SET
}

\author{
ASEEM DALAL AND PETER JOHNSON
}

\begin{abstract}
A set of vertices in a graph is perfect dominating if every vertex outside the set is adjacent to exactly one vertex in the set, and is neighborhood connected if the subgraph induced by its open neighborhood is connected. In any graph the full set of vertices is perfect dominating, and in every connected graph the full set of vertices is neighborhood connected. It is shown that

(i) in a connected graph, if the only neighborhood connected perfect dominating set is the full set of vertices, then the full set of vertices is also the only perfect dominating set; and (ii) if $r \geq 3$ and $n_{1}, \ldots, n_{r} \geq 2$, then in $K_{n_{1}, \ldots, n_{r}}$ the only perfect dominating set is the full set of vertices. Also, (iii) estimates are derived of how many edges can be removed from or added to $K_{n_{1}, \ldots, n_{r}}$ while preserving the property described in (ii).
\end{abstract}

\section{Introduction}

All graphs in this paper are finite and simple. The vertex set of a graph $G$ will be denoted $V(G)$, and the edge set will be denoted $E(G)$. If $u \epsilon V(G)$, the open neighborhood of $u$ in $G$, denoted $N_{G}(u)$, or $N(u)$, if $u$ is a vertex of no other graph than $G$ in the discussion, is $\{v \epsilon V(G) \mid u v \epsilon E(G)\}=\{v \epsilon V(G) \mid u$ and $v$ are adjacent in $G\}$. If $S \subseteq V(G), N(S)=\bigcup_{u \epsilon S} N(u)$, and $N[S]=S \cup N(S)$. The complement graph of $G$ is denoted by $\bar{G}$. Let $F$ be some set of edges, then $G \cup F$ is a graph formed by adding edges from the set $F$ to the graph $G$.

A set $S \subseteq V(G)$ is dominating in $G$ if and only if $V(G)=N[S]$; equivalently, each $w \epsilon V(G) \backslash S$ is adjacent to at least one vertex in $S$. The smallest size of a dominating set in $G$, the domination number of $G$, is denoted by $\gamma(G)$.

A set $S \subseteq V(G)$ is perfect dominating in $G$ if and only if each vertex $w \epsilon V(G) \backslash S$ is adjacent to exactly one $u \epsilon S$. Clearly $V(G)$ is perfect dominating in $G$. The smallest size of a perfect dominating set in $G$, the perfect domination number of $G$, is denoted by $\gamma_{p}(G)$.

A set $S \subseteq V(G)$ is neighborhood connected in $G$ if and only if $G[N(S)]$, the subgraph induced in $G$ by $N(S)$, is connected. Clearly $V(G)$ is neighborhood connected if and only if $G$

Received August 1, 2011, accepted January 30, 2013.

Communicated by Chung-Tsun Shieh.

2010 Mathematics Subject Classification. Primary 421305, 42B08.

Corresponding author: Aseem Dalal. 
minus its isolated vertices is connected, and is a neighborhood connected perfect dominating set in $G$ in that case. For $G$ connected, the smallest size of a neighborhood connected perfect dominating set will be denoted $\gamma_{n c p}(G)$.

A set $S \subseteq V(G)$ which is dominating (perfect dominating; neighborhood connected perfect dominating) with cardinality $\gamma(G)$ (resp., $\gamma_{p}(G) ; \gamma_{n c p}(G)$ ) is called a $\gamma(G)$-(resp., $\gamma_{p}(G)$-; $\left.\gamma_{n c p}(G)-\right)$ set. We are mainly concerned with a question raised in [3] : For which connected graphs $G$ is $\gamma_{n c p}(G)=|V(G)|$ ? That is, we are concerned with connected graphs $G$ in which no proper subset of $G$ is a neighborhood connected perfect dominating set. It will turn out that these are precisely the graphs of which the same is true with $\gamma_{p}$ replacing $\gamma_{n c p}$.

Perfect domination was introduced by P.M.Weichsel — see [2]. Neighborhood connected perfect domination was introduced in [3], evidently inspired by domination of another variety, neighborhood connected domination, introduced in [1].

\section{Results and Proofs}

Theorem 2.1. Suppose that $G$ is connected. Then $\gamma_{n c p}(G)=|V(G)|$ if and only if $\gamma_{p}(G)=$ $|V(G)|$.

Proof. Since, clearly, $\gamma_{n c p}(G) \geq \gamma_{p}(G)$, the "if" assertion is obvious. Suppose that $\gamma_{p}(G)<n=$ $|V(G)|$. We aim to show that $\gamma_{n c p}(G)<n$, i.e. , that there is a proper subset of $V(G)$ which is neighborhood connected and perfect dominating.

Let $S \subset V(G)$ be a perfect dominating set in $G$ and let $T=V(G) \backslash S \neq \phi$. Let $I=\{v \epsilon S \mid v$ is isolated in $G[S]$ \}. Because $S$ is dominating, $N(S)=V(G) \backslash I=(S \backslash I) \cup T$.

Because $G$ is connected, for every $w \epsilon S \backslash I$ there is a path in $G$ from $w$ to any vertex in $T$. Because $T \neq \phi$, there is a path with $w$ at one end, the other end in $T$, and every other vertex in $S \backslash I$. Consequently, if $G[T]$ is connected then $G[N(S)]$ is connected, so $S$ itself is a neighborhood connected perfect dominating set in $G$. We may assume that $G[T]$ is not connected. Let $H$ be one component of $G[T]$ and let $\tilde{S}=V(G) \backslash V(H) ; \tilde{S}$ is the union of $S$ with the vertex sets of the components of $G[T]$ other than $H$. Each $w \epsilon V(H)=V(G) \backslash \tilde{S}$ has exactly one neighbor in $\tilde{S}$, namely, the lone neighbor of $w$ in $S$. Therefore, $\tilde{S}$ is a perfect dominating set in $G$, a proper subset of $V(G)$, and is neighborhood connected because $H=G[V(G) \backslash \tilde{S}]$ is connected, by the argument above showing the analogous claim, for $S$, when $G[V(G) \backslash S]=$ $G[T]$ is connected.

The complete $r$-partite graph $G=K_{n_{1}, \ldots, n_{r}}$ is the graph with vertex set $V(G)=\bigcup_{i=1}^{r} P_{i}$ with $\left|P_{i}\right|=n_{i}$ for $1 \leq i \leq r$ (each set $P_{i}$ is called a part; the $P_{i}$ 's are pairwise disjoint) in which two vertices are joined if and only if they occur in different parts of $G$. In the case $r=1$ there is a 
notational conflict; we shall denote the complete 1-partite graph on $n_{1}$ vertices by $\overline{K_{n_{1}}}$, as is customary.

Remarks. It is easy to see that

(i) if $r=1$, then $\gamma_{p}\left(\overline{K_{n_{1}}}\right)=\gamma_{n c p}\left(\overline{K_{n_{1}}}\right)=|V(G)|$;

(ii) if $r=2, n_{1}=n_{2}=1$, then $\gamma_{p}\left(K_{n_{1}, n_{2}}\right)=\gamma_{n c p}\left(K_{n_{1}, n_{2}}\right)=1$;

(iii) if $r=2, n_{1}=1<n_{2}$ then $\gamma_{p}\left(K_{n_{1}, n_{2}}\right)=1$ and $\gamma_{n c p}\left(K_{n_{1}, n_{2}}\right)=2$;

(iv) if $r=2, n_{1}, n_{2} \geq 2$ then $\gamma_{p}\left(K_{n_{1}, n_{2}}\right)=\gamma_{n c p}\left(K_{n_{1}, n_{2}}\right)=2$;

(v) if $r \geq 3$ and $\min _{1 \leq i \leq r}\left\{n_{i}\right\}=1$, then $\gamma_{p}\left(K_{n_{1}, \ldots, n_{r}}\right)=\gamma_{n c p}\left(K_{n_{1}, \ldots, n_{r}}\right)=1$;

Theorem 2.2. Suppose $r \geq 3$ and $n_{1}, \ldots, n_{r} \geq 2$ are integers, and $G=K_{n_{1}, \ldots, n_{r}}$. Then $\gamma_{p}(G)=$ $\gamma_{n c p}(G)=|V(G)|$.

Proof. Suppose, on the contrary, that $\gamma_{p}(G)<|V(G)|$ and let $S \subseteq V(G)$ be a $\gamma_{p}(G)$ - set in $G$. . Since $V(G) \backslash S \neq \phi$, and $S$ is perfect dominating, without loss of generality we can assume the existence of $x \in P_{1} \backslash S$ such that $N(x) \cap S=\left(\bigcup_{j=2}^{r} P_{j}\right) \cap S=\{y\} \subseteq P_{2}$. Then $y$ is the sole member of $S$ adjacent to the vertices of $\cup_{j=3}^{r} P_{j}$. Consequently, $y$ is the only member of $S$. But then $S$ is not dominating, since $P_{2}$ contains a vertex other than $y$. This contradiction establishes that $\gamma_{p}(G)=\gamma_{n c p}(G)=|V(G)|$.

Finally, with $r \geq 3$ and $n_{1}, \ldots, n_{r} \geq 2$, we consider graphs obtained by removing edges from $K_{n_{1}, \ldots, n_{r}}$, and graphs obtained by adding edges to $K_{n_{1}, \ldots, n_{r}}$, and ask how many edges can be removed or added without changing the values of $\gamma_{p}$ and $\gamma_{n c p}$ from $\sum_{i=1}^{r} n_{i}$.

Theorem 2.3. Suppose that $r \geq 3,2 \leq n_{1} \leq \cdots \leq n_{r}$, and $\hat{G}=K_{n_{1}, \ldots, n_{r}}$, with parts $P_{i}$ oforder $n_{i}$, $i=1, \ldots, r$. The smallest size of a set $F \subseteq E(\hat{G})$ such that $G=\hat{G}-F$ is connected and $\gamma_{n c p}(G)<$ $|V(G)|$ is $n_{1}+\cdots+n_{r-2}$. Furthermore, the only such graphs $G=\hat{G}-F$, with $|F|$ minimum, have $\gamma_{n c p}(G)=2=\gamma_{p}(G)$, with all $\gamma_{n c p}$ - or $\gamma_{p}$ - sets consisting of two vertices, one from $P_{i}$, the other from $P_{j}$, for some $1 \leq i<j \leq r$ with $n_{i}, n_{j} \in\left\{n_{r-1}, n_{r}\right\}$.

Proof. If $1 \leq i<j \leq r, n_{i}, n_{j} \epsilon\left\{n_{r-1}, n_{r}\right\}$, and $u \epsilon P_{i}, v \epsilon P_{j}$, let $F \subseteq E(\hat{G})$ consist of one edge from each vertex of $V(G) \backslash\left(P_{i} \cup P_{j}\right)$ into $\{u, v\}$. Then $|F|=n_{1}+\cdots+n_{r-2}$ and $\{u, v\}$ is a $\gamma_{p}$ - and $\gamma_{n c p}$ - set in $G=\hat{G}-F$. (Because $n_{k} \geq 2$ for each $k$, no single vertex can be dominating in any spanning subgraph of $\hat{G}$.)

Let $n=\sum_{j=1}^{r} n_{j}$. Suppose that $F \subseteq E(\hat{G})$ is such that $G=\hat{G}-F$ is connected, with $\gamma_{p}(G)<$ $n$, and with $|F|$ minimum for such a set of vertices. By remarks above, $|F| \leq n_{1}+\cdots+n_{r-2}$. Let $S$ be a perfect dominating set in $G$ with $|S|<n$. Because $|F|$ is a minimum, any edge in $\hat{G}$ joining vertices of $S$, or joining vertices of $V(G) \backslash S$, is an edge of $G$. For $j \epsilon\{1, \ldots, r\}$, let $s_{j}=\left|S \cap P_{j}\right|$, and let $t=\left|\left\{i \mid s_{i}>0\right\}\right|$. 
Case I: $t=1$. If $t=1$ then, because $S$ is dominating in $G$, it must be that $S=P_{j}$ for some $j$. Since each vertex in $V(G) \backslash S$ is adjacent in $G$ to exactly one vertex in $S$, in this case we would have

$$
|F|=\left(n-n_{j}\right)\left(n_{j}-1\right)
$$

Since $n_{1} \leq n_{j} \leq n_{r} \leq n-\left(n_{1}+n_{2}\right)$, and $|F|$ is minimal, it must be that

$$
|F|=\left(n-n_{1}\right)\left(n_{1}-1\right) \geq n-n_{1}=n_{2}+\cdots+n_{r}>n_{1}+\cdots+n_{r-2},
$$

contrary to an earlier conclusion. Therefore $t>1$.

Case II: $t \geq 3$. Let $s=\sum_{j=1}^{r} s_{j}$. Suppose that $s_{j}>0$ for some $1 \leq j \leq r$. Pick any $u \epsilon S \cap P_{j}$. We will make another graph $G^{\prime}=\hat{G}-F^{\prime}$, for some $F^{\prime} \subseteq E(\hat{G})$, in which $S^{\prime}=S \backslash\{u\}$ is a perfect dominating set. We make $G^{\prime}$ by adding and subtracting edges of $\hat{G}$ to and from $G$, as follows.

1. For each $v \in V(G) \backslash S$ which is adjacent, in $G$, to $u$ (and therefore only to $u$, among vertices in $S$ ), delete the edge $u v$ and make $v$ newly adjacent to some other vertex in $S$, not in $v$ 's part. Since $t \geq 3$, there will be a vertex of $S$ in a part different from the parts of $u$ and $v$.

2. Delete all edges from $u$ to other members of $S$, except one (and there will be one, because $t>1)$, and add in all edges from $u$ to $\left(V(G) \backslash P_{j}\right) \backslash S$, including any that might have been replaced in step 1 .

It is straight forward to see that $S \backslash\{u\}$ is a perfect dominating set in $G^{\prime}$, and that $G^{\prime}=\hat{G}-F^{\prime}$ where $F^{\prime} \subseteq E\left(G^{\prime}\right)$ satisfies

$$
\left|F^{\prime}\right|=|F|+\left(s-s_{j}-1\right)-\left[n-n_{j}-\left(s-s_{j}\right)\right]=|F|+\left[2 s-n-\left(2 s_{j}-n_{j}\right)-1\right] .
$$

Because all $\hat{G}$ edges between vertices in $S^{\prime}$ are edges of $G^{\prime}$, and $S^{\prime}$ has representatives in at least two of the $P_{i}, G^{\prime}\left[S^{\prime}\right]$ is connected. Therefore, because $S^{\prime}$ is perfect dominating in $G^{\prime}$, it follows that $G^{\prime}$ is connected. By the minimality of $|F|$, it follows that $2 s-n-\left(2 s_{j}-n_{j}\right)-1 \geq 0$.

Now suppose that $0<s_{j}<n_{j}$ and take any $w \epsilon P_{j} \backslash S$. We will produce a graph $G^{\prime \prime}=\hat{G}-F^{\prime \prime}$, for some $F^{\prime \prime} \subseteq E(\hat{G})$ in which $S \bigcup\{w\}=S^{\prime \prime}$ is a perfect dominating set. To form $G^{\prime \prime}$, add to $G$ the $s-s_{j}-1$ edges from $w$ to $S \backslash P_{j}$ that were not in $G$, and delete from $G$ the $n-n_{j}-\left(s-s_{j}\right)$ edges from $w$ to $V(G) \backslash S$ that were in $G$. It is clear that $S^{\prime \prime}$ is perfect dominating in $G^{\prime \prime}$ and that $G^{\prime \prime}$ is connected, by an argument similar to that for $G^{\prime}$. Also, $\gamma_{p}\left(G^{\prime \prime}\right)<n$, for, if not, then $S^{\prime \prime}=V\left(G^{\prime \prime}\right)=V(\hat{G})$, so $S=V(\hat{G}) \backslash\{w\}$; but then

$$
|F|=n-n_{j}-1>n_{1}+\cdots+n_{r-2} .
$$

We have $G^{\prime \prime}=\hat{G}-F^{\prime \prime}$ with

$$
\left|F^{\prime \prime}\right|=|F|+n-n_{j}-\left(s-s_{j}\right)-\left(s-s_{j}-1\right)=|F|-\left[2 s-n-\left(2 s_{j}-n_{j}\right)-1\right]
$$


By the minimality of $|F|$, it must be that $2 s-n-\left(2 s_{j}-n_{j}\right)-1 \leq 0$. We conclude that if $t \geq 3$ and $1 \leq s_{j}<n_{j}$ then $2 s-n-\left(2 s_{j}-n_{j}\right)-1=0$, and we can find $G^{\prime \prime}=\hat{G}-F^{\prime \prime}$, as above, with $|F|=\left|F^{\prime \prime}\right|$ and a perfect dominating set $S^{\prime \prime}$ with $\left|S^{\prime \prime}\right|=|S|+1<n ; S^{\prime \prime}$ has one more vertex in $P_{j}$ than does $\mathrm{S}$.

Consequently, if $t \geq 3$ we can modify $G$ and $S$ successively, so that we may as well assume that $S$ is a union of $t$ of the $P_{j}$; it must be that $t<r$, because $|S|<n$. We have that

$$
|F|=(n-s)(s-1)
$$

Since $s$ is a sum of 3 or more of the $n_{j}$, and $s<n$, we have $n_{1}+n_{2}+n_{3} \leq s \leq n-n_{1}$. Therefore,

$$
\begin{aligned}
|F| & =(n-s)(s-1) \geq\left(n-\left(n-n_{1}\right)\right)\left(n-n_{1}-1\right)=n_{1}\left(n-n_{1}-1\right) \\
& >n_{1}\left(n-n_{1}-n_{2}\right)>n_{3}+\cdots+n_{r} \geq n_{1}+\cdots+n_{r-2}
\end{aligned}
$$

contradicting previous findings.

Case III: $t=2$. Suppose that $s_{i}, s_{j}>0, i \neq j$. Then,

$$
\begin{aligned}
n-\left(n_{r}+n_{r-1}\right) & =n_{1}+\cdots+n_{r-2} \geq|F| \\
& \geq\left(n-n_{i}-n_{j}\right)(s-1)+\left(s_{i}-1\right)\left(n_{j}-s_{j}\right)+\left(s_{j}-1\right)\left(n_{i}-s_{i}\right) \\
& \geq\left(n-n_{i}-n_{j}\right)(s-1) \geq\left(n-n_{r}-n_{r-1}\right)(s-1) \geq n-n_{r}-n_{r-1}
\end{aligned}
$$

It follows that $s=2, s_{i}=s_{j}=1$, and $n_{i}, n_{j} \epsilon\left\{n_{r-1}, n_{r}\right\}$.

Theorem 2.4. Suppose that the hypotheses of Theorem 2.3 hold. The smallest size of a set $F \subseteq$ $E(\overline{\hat{G}})$ such that, if $G=\hat{G} \cup F$, then $\gamma_{p}(G)<|V(G)|$, is $n_{1}-1$. Further, the only such $G$ are obtained by joining some vertex in some $P_{j}$ such that $n_{j}=n_{1}$ to its $n_{1}-1$ companions in $P_{j}$.

Proof. Since adding all the edges of its complement to $\hat{G}$ yield a complete graph, $K_{n}, n=$ $n_{1}+\cdots+n_{r}$, which has $\gamma_{p}=\gamma_{n c p}=1$, there must be some minimum-sized set $F \subseteq E(\overline{\hat{G}})$ such that $G=\hat{G} \cup F$ has $\gamma_{p}(G)<n$. Let $F$ be such a set. Let $S$ be a perfect dominating set in $G$ with $|S|<n$.Because all the edges of $\hat{G}$ are edges of $G$, if $S$ has two elements $u, v$, then every $P_{j}$ containing neither $u$ nor $v$ would have to be contained entirely in $S$. Since $r \geq 3$ there would be such a $P_{j}$; and since $n_{j} \geq 2$ every $P_{i} \subseteq S$. Therefore, since $|S|<n,|S|=1$. For $S$ to be dominating in $G$, the single vertex in $S$ must be adjacent to every vertex in its part, and making this so will suffice to make $\gamma_{p}(G)=\gamma_{n c p}(G)=1<n$. Clearly $n_{1}-1$ is the minimum number of edges necessary to bring this about. 


\section{References}

[1] S. Arumugam and C. Sivagnanam, Neighborhood connected domination in graphs, J. Combin. Math. Combin. Comput., 73(2010), 55-64.

[2] T. W. Haynes, S. T. Hedefniemi and P. J. Slater, Fundamentals of Domination in Graphs, Marcel Dekker, Inc, New York, 1997.

[3] P. Selvaraju, M. P. Kulaindaivel and C. Sivagnanam, Neighborhood connected perfect domination in graphs, Tamkang Journal Of Mathematics, 43(2012), 557-562.

Department of Mathematics, Indian Institute of Technology, Delhi-110016 , India.

E-mail: aseem.dalal@gmail.com

Department of Mathematics and Statistics, Auburn University, AL 36849, USA.

E-mail: johnspd@auburn.edu 